\title{
GRUPO DE ORIENTAÇÃO CLÍNICO-NUTRICIONAL A FAMILIARES DE PORTADORES DE TRANSTORNOS ALIMENTARES: UMA EXPERIÊNCIA “GRATA"*
}

\author{
GROUP OF CLINICAL-NUTRITIONAL ORIENTATION TO THE FAMILY OF BEARERS OF \\ ALIMENTARY DISORDERS THE "GRATA" EXPERIENCE
}

Felícia Bighetti ${ }^{1}$, José Ernesto Dos Santos², Rosane Pilot Pessa Ribeiro³

\begin{abstract}
1Docente. Universidade de Franca - UNIFRAN. ${ }^{2}$ Docente. Divisão de Nutrologia. Departamento de Clinica Médica. Faculdade de Medicina de Ribeirão Preto - USP. ${ }^{3}$ Docente. Departamento de Enfermagem Materno-Infantil e Saúde Pública. Escola de Enfermagem de Ribeirão Preto - USP

CorRespondência: Felícia Bighetti. Rua: José Bonini, 1415, Centro. CEP 14.160.160. Sertãozinho/SP
\end{abstract}

Bighetti F, Dos Santos JE, Ribeiro RPP. Grupo de orientação clínico-nutricional a familiares de portadores de transtornos alimentares: Uma experiência GRATA. Medicina (Ribeirão Preto) 2006; 39 (3): 410-4

RESUMO: O tratamento dos transtornos alimentares deve envolver múltiplas abordagens do ponto de vista clínico, nutricional, psicológico e psiquiátrico desenvolvidas por uma equipe multi e interdisciplinar. Nesse contexto, o GRATA, ao longo de 20 anos como serviço especializado na assistência dessas doenças, vem se aprimorando e crescendo com o número de profissionais, o que possibilitou ampliar as modalidades de intervenção, oferecendo apoio e orientação para os familiares, além do próprio portador. Para tanto, organizou um grupo de pais e acompanhantes que se dividem em dois momentos: o primeiro coordenado por uma nutricionista e um médico, e o segundo coordenado por duas psicólogas, caracterizados como grupos abertos, realizados semanalmente com duração de uma hora cada e no dia do atendimento ambulatorial.

Nesses quatro anos de experiência, evidenciou-se melhor adesão do paciente e família ao tratamento como um todo, com comparecimento médio de 8 pessoas por grupo. Na visão clíniconutricional, percebeu-se evolução mais favorável, além do reconhecimento e gratidão expressados ao se sentirem também cuidados. Por outro lado, a exigência de investimentos no bem-estar mental dos seus profissionais é de extrema importância e se dá por meio de treinamentos e incentivo ao resgate de recursos internos dos coordenadores e da equipe como um todo, que se reúne semanalmente para supervisão dos grupos, dos casos atendidos individualmente e da própria equipe. Apesar dos obstáculos enfrentados, inerentes a um tratamento tão complexo e desafiador, esses diversos olhares dos profissionais envolvidos têm possibilitado uma GRATA experiência.

Descritores: Transtornos da Alimentação. Assistência Familiar. Equipe Multidisciplinar.

Os transtornos alimentares, entre eles a anorexia nervosa $(\mathrm{AN})$ e bulimia nervosa $(\mathrm{BN})$, afetam principalmente jovens do sexo feminino na idade entre 12 e 18 anos, apresentando elevada prevalência não só nos países desenvolvidos, onde subsistem as características econômicas e socioculturais para seu desencadeamento, como também nos países de terceiro mundo ${ }^{1,2,3}$.

\footnotetext{
* Grupo de Assistência em Transtornos Alimentares (GRATA) do Hospital das Clínicas da Faculdade de Medicina de Ribeirão Preto - USP (HCFMRP-USP)
} 
Os sintomas desses quadros abrangem áreas bastante diversas, com perturbações nutricionais, endocrinológicas, cognitivas e psicodinâmicas, ou seja, tanto o funcionamento psíquico quanto o somático estão alterados.

Diante dessa natureza ampla e complexa requerem tratamento igualmente complexo. Atualmente, o trabalho em equipe multidisciplinar tem sido reconhecido como a forma mais adequada de tratamento. A estrutura básica de uma equipe multidisciplinar para o tratamento dos transtornos alimentares deve ser composta por psiquiatra, nutricionista, psicólogo, terapeuta familiar e clínico geral ou médico nutrológo. No entanto, outros profissionais como endocrinologistas, terapeuta ocupacional, enfermeiro etc., também podem fazer parte da equipe e contribuir de maneira importante 4 .

Nunes et al. (1998) $)^{5}$ enfatizam que todo esse tratamento de origem multidisciplinar deve ser realizado e estabelecido para todos os pacientes como de importância extrema, cabendo aos membros da equipe buscar maneiras criativas para atração tanto desses como dos familiares para fazer com que não haja, assim, tanta dificuldade no tratamento e conseqüente baixa adesão e negação da doença, o que é de bastante freqüência nos serviços de assistência.

Existem várias teorias e técnicas de intervenção familiar desenvolvidas para o tratamento dos transtornos alimentares. A maioria dessas intervenções foi estudada e implantada para famílias de anoréxicos, embora existam também estudos realizados com portadores de pacientes com bulimia nervosa.

Essas intervenções propõem atuar nos fatores mantenedores dos transtornos alimentares, ou seja, na dificuldade da família no manejo dos conflitos, na superproteção parental, no paciente como papel de "bode expiatório" e na alta emoção expressa. Podem ser realizadas intervenções em grupos de familiares ou de pais de pacientes, intervenções nas famílias de pacientes com anorexia nervosa ou bulimia nervosa ou a terapia familiar propriamente dita ${ }^{6}$.

$\mathrm{O}$ tratamento no grupo de pais tem um formato em que o paciente não comparece ao grupo, apenas seus familiares ou acompanhantes. As reuniões são, em geral, semanais e podem durar todo o tempo de tratamento do paciente. Têm uma proposta psicoeducacional, de orientação aos pais e amigos de como lidarem com o membro doente. Nesse modelo, o indivíduo doente, e não a dinâmica familiar, é o alvo do tratamento.
Ainda, o mesmo autor refere que é preciso observar que a terapia familiar está longe de ser um campo unificado de intervenções e teorias, mas a base desse tipo de intervenção é o entendimento de que a doença é parte do sistema e nele tem uma função. A proposta da intervenção familiar é a de poder avaliar esse sistema, educá-lo quanto à doença e tratá-lo caso a avaliação de sua dinâmica indique um funcionamento que promova a manutenção do sintoma ${ }^{6}$.

\section{A experiência do GRATA no atendimento em grupo para apoio aos familiares}

O GRATA - Grupo de Assistência aos Transtornos Alimentares, é um programa do Ambulatório de Nutrologia do Hospital das Clínicas da Faculdade de Medicina de Ribeirão Preto-USP, que existe desde 1982.

O intuito inicial foi oferecer tratamento para os primeiros casos de transtornos alimentares que chegaram em busca de assistência. Modestamente, foi composto por um nutricionista e um nutrólogo fortemente influenciados por um psiquiatra, na época médico assistente contratado pela Divisão de Nutrologia que teve a iniciativa de organizar as rotinas do serviço. Contava assim, com poucos profissionais que tinham a curiosidade de tentar atender os desconhecidos anoréxicos e bulímicos e propor estratégias terapêuticas multidisciplinares.

Uma experiência pioneira com grupo de familiares de portadores de transtornos alimentares se deu quando Bó e Barbosa (1999) ${ }^{7}$, na época psiquiatras voluntários, se integraram ao serviço, já que não existia um trabalho sistematizado com os familiares de pacientes.

Bó e Barbosa $(1999)^{7}$, conceberam um grupo de múltiplas famílias do tipo dos de sala de espera, no qual participavam os familiares que acompanhavam os pacientes ao ambulatório, enquanto estes eram atendidos em suas consultas com o médico e/ou nutricionista. A característica básica do grupo era aberto, com o comparecimento de seus membros vinculado ao retorno dos pacientes às consultas, a freqüência das sessões era semanal com uma média de 4 a 5 participantes e estes eram geralmente mães. Desempenhavam, respectivamente, as funções de coordenador e observador. Durante o trabalho grupal, no qual a troca de experiências era fundamental, a repetição inconsciente dos comportamentos cotidianos era confrontada e então, novas formas de agir e pensar poderiam ser aprendi- 
das, transformando os indivíduos. Todos eram responsáveis pelo resultado final da interação grupal e as diferenças eram respeitadas e vistas como enriquecedoras. Concluíram assim, que o grupo de múltiplas famílias, além dos resultados terapêuticos já descritos, pode ser muito útil na inclusão e envolvimento das famílias no tratamento dos transtornos alimentares.

Após essa experiência, a equipe ampliou-se ainda mais com a inserção de um psicólogo e atualmente, é composta por cerca de 25 pessoas, entre alunos e profissionais, médicos nutrólogos, nutricionistas, psicólogos, psiquiatras, além de enfermeiro e terapeuta ocupacional na modalidade de internação. Oferece, assim, tratamento especializado com atendimento individual e em grupo para o paciente e sua família. É responsável ainda pela capacitação de residentes, pósgraduandos e graduandos, que têm a rica e privilegiada oportunidade de aprender sobre essas graves síndromes.

Os avanços alcançados com as abordagens mais eficazes foram muitos, mas o espírito do grupo é sempre de busca por novas possibilidades terapêuticas. Desde então, este serviço vem se aprimorando e crescendo com o número de profissionais especializados para prestações de serviços e pesquisas.

No decorrer desse caminho de importantes conquistas, há cerca de 4 anos, houve a elaboração de atendimentos em grupos para apoiar familiares, afim de conhecê-los, orientá-los e proporcionar trocas entre eles e os profissionais, devido à crescente demanda que o serviço foi recebendo.

Justifica-se ainda o fato de que essas doenças são psicossomáticas, ou seja, de origem emocional e orgânica, o que gera nos familiares muita angústia, por não saberem lidar com tal desafio.

Esse grupo de apoio a familiares em novo formato existe desde 2001, possibilitando aos profissionais que atuam no serviço uma visão mais profunda de como funciona essa dinâmica familiar e também acolhendo melhores esses familiares.

Nessa perspectiva, foram formados dois grupos, sendo que o primeiro é coordenado atualmente por uma nutricionista e um médico, e o segundo coordenado por duas psicólogas.

Caracterizam-se por serem grupos abertos e realizados semanalmente, em sequiência, no dia de atendimento ambulatorial com duração de uma hora cada.

O grupo coordenado pela nutricionista e médico tem perfil informativo e educativo cujos objetivos gerais que norteiam as intervenções são:
1) promover noções sobre os transtornos alimentares, suas implicações físicas, psicológicas, familiares e sociais, e modalidades do tratamento;

2) prover informações sobre nutrição do organismo, desnutrição e seu quadro fisiopatológico, sinais clínicos dessas síndromes e suas conseqüências metabólicas;

3) esclarecer os familiares sobre as orientações alimentares que os pacientes recebem e auxiliá-los para os seguimentos destas em domicílio;

4) orientar os familiares quanto à melhor conduta frente ao que os pacientes apresentam em relação ao comportamento alimentar e práticas inadequadas relacionadas à seleção, compra e manuseio dos alimentos;

5) discutir assuntos trazidos espontaneamente pelo grupo (puberdade, adolescência, relacionamento entre pais e filhos, manejo da alimentação da família, etc).

Os resultados desse trabalho evidenciam a melhor adesão do paciente e família ao tratamento, cujos benefícios são revelados à medida que a participação torna-se efetiva e mantenedora.

Apesar dessas evidentes melhoras, também são acolhidas as queixas que são constantemente presentes nas falas dos familiares, principalmente com relação ao funcionamento do serviço: demora no atendimento, a longa distância de suas cidades até o serviço e o tempo de viagem, o atendimento ambulatorial junto ao ambulatório de pacientes obesos graves, incomodando muito os familiares e pacientes.

Esse fato reflete na resistência inconsciente desses familiares ao aceitarem suas próprias limitações, angústias, dificuldades dos conflitos e problemas mal resolvidos entre todos no ambiente domiciliar.

O trabalho grupal traz o esclarecimento de malentendidos, o respeito da individualidade de cada um, possibilitando-lhes um aprendizado que pode ser transferido ao ambiente familiar.

Nesses quatro anos de experiência de atendimentos em grupos a estes familiares, observou-se que o comparecimento se faz, na maioria das vezes, pelos pais, irmãos, esposos, avós ou outros acompanhantes com média de 8 pessoas por grupo, participação esse maior que a primeira iniciativa feita por Bó e Barbosa (1999) ${ }^{7}$.

A princípio, os familiares mostram-se resistentes em aceitar a doença e apreensivos em entenderem os sintomas e comportamentos que assumem frente à alimentação e à doença como um todo. Chegam 
ao grupo em movimento voraz com desejo de resolverem tais angústias de forma rápida e precisa; trazem dúvidas de o quê vem a ser a doença, sua incidência e principalmente estatísticas de curas. Nesse sentido, o grupo tem um perfil bastante acolhedor e realista ao informar que os resultados são variáveis e que a evolução depende de como cada um (paciente e família) se envolve e reage ao tratamento.

Por meio da convivência grupal e a medida em que vão tendo maior frequiência nos grupos, os participantes entram mais em contato com suas dores, expectativas, melhoras, reconhecem suas próprias dificuldades e aprendem a construir seu saber pelas trocas da vivência de cada um ali presente.

Na visão clínico-nutricional, percebe-se evolução favorável de cada um desses familiares e a gratidão de estarem também recebendo cuidados.

A equipe reúne-se semanalmente para supervisão dos grupos e da própria equipe, além de outras discussões teóricas. Durante o encontro, os dados desses familiares são analisados juntamente com as informações colhidas pelos outros profissionais que realizam atendimentos individuais com os pacientes e familiares, favorecendo maior entendimento dessas famílias e colaborando para estratégias terapêuticas de seus respectivos doentes anoréxicos e bulímicos.
O GRATA tem crescido e se solidificado muito com essas experiências, que foram e estão sendo de extremo valor e importância tanto para a equipe quanto para o paciente e familiar.

Isto reflete na exigência de investimentos no bem-estar mental da equipe, por meio de treinamentos e apoio. Um deles, o Instituto Familiae, que é representado por um grupo de profissionais, a maioria psicólogos, oferece exercícios dinâmicos para a equipe poder trabalhar suas angústias, comunicação e outras, dificuldades internas entre os próprios membros e também no atendimento de familiares e pacientes. O trabalho de cuidar de quem cuida traz reflexões da prática clínica e contribui efetivamente para a equipe lidar com suas frustrações, limites e desafios.

Todos esses conhecimentos e conquistas que a equipe tem alcançado também é fruto da divulgação das atividades em eventos e intercâmbio com outros serviços que oferecem tratamento integrado e especializado $^{8,9}$.

Conclui-se portanto, que essa GRATA experiência e os diversos olhares dos profissionais presentes tem promovido influências harmônicas o bastante para uma satisfatória evolução para a maioria dos pacientes que necessitam de tratamento tão complexo e desafiador.

Bighetti F, Dos Santos JE, Ribeiro RPP. Group of clinical-nutritional orientation to the family of bearers of alimentary disorders. The GRATA experience. Medicina (Ribeirão Preto) 2006;39 (3): 410-4.

ABSTRACT: The treatment of the alimentary problems should involve multiple approaches from the clinical, nutritional, psychological and psychiatric point of view developed by a multi and interdisciplinary team. In this context, GRATA, along 20 years as a specialized service in the assistance of these diseases, is improving and growing with the number of professionals, what enabled the increasing of the intervention modalities, offering support and orientation for the family, besides the own bearer. To do so, it organized a group of parents and companions that is divided into two moments: the first coordinated by a nutritionist and a resident of Medicine, and the second coordinated by two psychologists, characterized as opened groups, held weekly and lasting one hour each and in the day of the assistance.

In these four years of experience, it was evidenced a better adhesion of patient and family to the treatment as a whole, with average attendance of 8 people in a group. In the clinical-nutritional vision, a more favorable evolution was noticed, besides the recognition and gratitude expressed when they also feel cared. On the other hand, the demand of investments in the mental welfare of its professionals is of extreme importance and happens by means of training and incentive to the collecting of internal resources of the coordinators and of the team as a whole, which get together weekly for the supervision of the groups, of the cases attended individually and of the team. Despite the obstacles faced, inherent to such a complex and challenger treatment, these several views of professionals involved have enabled a Thankful (GRATA) experience.

Keywords: Eating Disorders. Family Assistance. Multidisciplinary Team. 


\section{REFERÊNCIAS}

1 - Lucas AR, Beard CM, O'Fallon WN, Kurland LT. 50-year trends in the incidence of anorexia nervosa in Rochester, Minnesota: population - based - study. Am J Psychiatry 1991; 148: 917-22

2 - Oyewumi LK, Kazarian SS. Abnormal eating attitudes among a group of nigerian youths: II Anorexic behaviour. East Afr Med J 1992; 69: 667-9

3 - Hoek HM. Review of the epidemiological studies of eating disorders. Int Rev Psychiatry 1993; 5: 61-74

4 - Sapoznik A, Bueno MO, Lobão BF. Tratamento - Aspectos gerais. In: Claudino AM, Zanella MT. Transtornos alimentares e obesidade. São Paulo: Manole; 2005. p 111-8.

5 - Nunes MAA, Appolinário JC, Abuchaim, ALG, Coutinho W, eds. Transtornos alimentares e obesidade. Porto Alegre: Artmed, 1998.
6 - Stefano SC, Passos TCP, Kern LK. Abordagens Psicológicas. In: Claudino AM, Zanella MT. Transtornos alimentares e obesidade. São Paulo: Manole; 2005. p. 137-46.

7 - Bó DEB, Barbosa R. Anorexia, bulimia e família: uma experiência com trabalho em grupo. J Bras Psiquiatr 1999; 48: 533-7.

8 - Bighetti F, Santos RK, Mouraria CC, Soares IVB, Prizanteli CC, Godoy RSP, Ribeiro RPP, Santos MA. Grupo de apoio psicológico aos familiares de portadores de anorexia e bulimia nervosa. Rev SPAGESP 2002; n. 3 esp: 139-43.

9 -Bighetti F. Grupo de Orientação Clínico-nutricional. In: Anais do II Simpósio sobre transtornos alimentares - construindo um modelo assistencial - Grupo de Assistência em Transtornos Alimentares da Faculdade de Medicina do Hospital das Clínicas de Ribeirão Preto da USP (GRATA), Ribeirão Preto; 2005. p. 31 